\title{
Analysis of Min-Trees over Sentinel-1 Time Series for Flood Detection
}

\author{
Çaglayan Tuna \\ Université Bretagne Sud, IRISA \\ Vannes, France \\ caglayan.tuna@irisa.fr
}

\author{
François Merciol \\ Université Bretagne Sud, IRISA \\ Vannes, France \\ francois.merciol@irisa.fr
}

\author{
Sébastien Lefèvre \\ Université Bretagne Sud, IRISA \\ Vannes, France \\ sebastien.lefevre@irisa.fr
}

\begin{abstract}
Monitoring flood is an important task for disaster management. It requires to distinguish between changes related to water from the other changes. We address such an issue by relying on both spatial and intensity information. To do so, we exploit min-tree that emphasize intensity extrema in a multiscale, efficient framework. We thus suggest a two-step approach operating on satellite image time series. We first perform a temporal analysis to identify images containing possible floods. Then a spatial analysis is achieved to detect flood areas on the selected images. Both steps relies on the analysis of component attributes extracted from the min-tree representation. We conduct some experiments on a flooded scene observed through Sentinel-1 SAR imagery. The results show that flood areas can be efficiently and accurately characterized with spatial component attributes extracted from hierarchical representations from SAR time series.
\end{abstract}

Index Terms-Satellite Image Time Series, Flood mapping, Sentinel-1, Hierarchical representations

\section{INTRODUCTION}

Thanks to their high revisiting ability, Sentinel missions are suitable tools for short-term Earth Observation. Among their possible usages, change detection receives a significant attention since it offers various applications, e.g. urban area monitoring, disaster management, etc. Flood is a frequent disaster that requires forecasting, monitoring, and assessment. Synthetic Aperture Radar (SAR) has established as a relevant data source for change detection [1], as well as flood mapping [2], [3]. In this paper, we propose to rely on a multiscale, hierarchical representation of the SAR image time series to extract and characterize flooded areas.

Indeed, morphological hierarchies through tree-based representations are now established as an efficient way to implement (spatially) connected filters [4] over ordered sets such as grayscale images. They have especially been successful in remote sensing, e.g. for classification [5] or segmentation [6]. Extension of such hierarchies to satellite image time series (SITS) has only been addressed in very few works. AlonsoGonzález et al. propose in [7] to build a Binary Partition Tree between two SAR images considering a temporal connectivity. Falco et al. perform pixelwise change detection [8] comparing sums of differential attribute profiles (AP) in each pixel. Recently, monitoring urban growth was achieved in [9] where spatial area attributes of the SITS are derived from hierarchical representations. However, and to the best of the authors' knowledge, such hierarchical representations were never used for flood detection yet.

The SAR backscatter depends on the physical properties of the objects, with water reflecting less than other materials [3]. Therefore, radiometric thresholding is known as an efficient way to extract water areas [10]. However, such a method is very sensitive to noise and spatial regularization thus appears as a relevant strategy to improve robustness. To do so, we propose here to rely on morphological representations and characterize dark connected components using various attributes such as level, area and stability. While bright object detection (ship) was performed with max-tree in [11], we focus here on dark object and thus use rather the min-tree. We rely on multiple representations of a SITS: first, we measure the intrinsic complexity of each image based on a spatial hierarchy to identify dates where floods occur; then, we build a spatiotemporal hierarchy from both flood and non-flood images in order to spatially delineate the flood footprint. Experimental assessment and comparison with related works shows the relevance of our approach.

The paper is organized as follows. We first present our dataset in Sec. II before introducing our proposed method to detect flood in Sec. III. We then report experimental results in Sec. IV before concluding the paper with Sec. V.

\section{DATA}

We illustrate our approach with Sentinel-1A images acquired over Montmirail, North of France and East of Paris. All images come with a $10 \mathrm{~m}$ resolution Ground Range Detected products with the Interferometric Wide Swath (IW) mode. The SITS is made of 3 images acquired on 17 July 2017, 10 August 2017 and 25 January 2018. We rely on the Copernicus Emergency Management Service flood mapping ${ }^{1}$ shape files as a ground truth for assessing our method. The flood occurred on 22 January 2018, i.e. between second and third images of the series.

For the sake of illustration, we focus on two specific areas where floods occurred, as shown in Fig. 11 (histogram equalization is performed solely for visibility) that also contains reference flood maps. The two SITS samples are made of $1276 \times 2803$ and $892 \times 1941$ pixels, respectively. A close-up

\footnotetext{
${ }^{1}$ https://emergency.copernicus.eu/
} 
view is provided in Fig. 2 to ease visual assessment of the changes, and distinguishing changes due to floods from other changes (e.g. related to crops).

\section{Proposed Method}

In order to increase noise robustness, we propose to rely on the spatial connectivity of the water pixels. Such information is efficiently captured with hierarchical representations that allow to extract meaningful regions or components and to describe them with some spatial or intensity attributes.

In a nutshell, our pipeline is made of three main steps. We first coregister and calibrate the input images. Pixel values are then normalized from float values as 16-bit integers to ease computation. The two next steps, namely temporal and spatial detection, are described in the following subsections.

\section{A. Temporal detection}

We first aim to identify which images of the SITS are most likely to contain floods. This process can be seen as a specific case of change detection, where only water-related changes are sought. To do so, we focus on dark areas in the image that correspond to water pixels. These areas form leaves in the min-tree image representation, that is built from connected components of the lower level sets of an image.

More formally, for a grayscale image $I: \Omega \in \mathbb{N}^{2} \rightarrow V \in \mathbb{Z}$, $x \mapsto I(x)=v$ with $x$ and $v$ denoting coordinates and associated intensity values respectively, we define the lower threshold set $L_{\lambda}(I)$ as $[I \leq \lambda]=\{x \in \Omega, I(x) \leq \lambda\}$ with a threshold $\lambda \in \mathbb{Z}$. The lower level set is then $L_{\lambda}(I)=$ $C C([I \leq \lambda])=\{x \in \Omega \mid C C(I(x) \leq \lambda)\}$ where $C C(X)$ denotes the set of connected components of $X$ (we consider here 4-connectivity). A tree $\mathcal{T}$ is then defined as the set of all nodes $\mathcal{N}$ or $\left\{L_{\lambda}(I)\right\}_{\lambda \in \mathbb{R}}$.

In order to find which image is most likely to contain floods in the time series, we characterize each image $I_{t}$ by some measures computed from the nodes $\mathcal{N}$ from its corresponding tree $\mathcal{T}\left(I_{t}\right)$. Indeed, we assume that the successive images of a scene share the same topology and contain similar spatial structures. Although intensity is changing (see Fig. 2) during time, the shapes remain similar. The min-tree offers an efficient way to analyze such structural information through time.

We consider here two different measures to characterize the structural complexity of an image. The first, straightforward measure is simply the amount of nodes contained in the tree $\mathcal{T}\left(I_{t}\right)$. Another, more advanced feature is based on the concept of stability used in the Maximally Stable Extremal Regions (MSER). MSER relies on the growing rate of the nodes along a tree branch [12]:

$$
s(\mathcal{N})=\frac{A(\mathcal{N})-A\left(\mathcal{N}_{\triangle}\right)}{A(\mathcal{N})}
$$

where $A$ represents the area of the relevant node $\mathcal{N}, \triangle$ is a predefined distance between $\mathcal{N}$ and its ancestor node, and $s$ is the stability. We set here $\triangle=5$. Stable nodes are characterized by a significant stability value, so we choose here $s \geq 0.001$. The number of stable regions is then used as a complexity indicator.
Table \illustrates these two measures for the successive images of the two SITS. We can see that both are good indicators of the image complexity. More stable regions indicate that the corresponding image includes more shapes or structures. It actually happens at time $t=3$, i.e. in case of a flood (see Sec. III). Once the flood event date has been identified, we use related images $I_{3}$ and $I_{3}^{\prime}$ as flooded images among their respective SITS. We then compare these images with other dates to achieve a spatial delineation of the floods, as described in the next subsection.

\begin{tabular}{c|c|c} 
& \# Nodes & \# MSER \\
\hline$I_{1}$ & $1,376,403$ & 30,699 \\
\hline$I_{2}$ & $1,486,696$ & 34,400 \\
\hline$I_{3}$ & $1,531,558$ & 37,881
\end{tabular}

\begin{tabular}{c|c|c} 
& \# Nodes & \# MSER \\
\hline$I_{1}^{\prime}$ & 789,554 & 20,607 \\
\hline$I_{2}^{\prime}$ & 835,335 & 24,853 \\
\hline$I_{3}^{\prime}$ & 859,766 & 29,513
\end{tabular}

TABLE I: Evolution of structural complexity for $I$ and $I^{\prime}$.

\section{B. Spatial detection}

As already noticed, water pixels are dark in SAR imagery, and their intensity values are lower than for other materials. Thresholding backscatter values is a very common strategy for flood mapping [10]. However, it is very sensitive to noise. We propose to use another min-tree representation to improve the detection robustness.

More precisely, we consider two successive images from the time series, respectively before and after the flood occur, i.e. in our case $\left(I_{2}, I_{3}\right)$ and $\left(I_{2}^{\prime}, I_{3}^{\prime}\right)$. We now build a spatio-temporal min-tree where the connectivity is both in space and time domains (i.e. 6-connectivity). Within this spatio-temporal tree, we explore the relationship between parent and child nodes for each level of the tree. Since the nodes embed now some temporal information, the variability of intensity values helps to locate where the intensity values are dramatically changing. It gives us valuable insights for spatially delineating the flood areas.

We thus measure for each node $\mathcal{N}$ the difference in variances between the node and its children:

$$
V(\mathcal{N})=\sum_{i}\left(\operatorname{Var}(\mathcal{N})-\operatorname{Var}\left(\mathcal{N}_{c}^{i}\right)\right)
$$

with $\mathcal{N}_{c}$ the children nodes of $\mathcal{N}$ and $i$ their index. We then sum these values for each level $\lambda$ of the tree, leading to a global variance difference measure defined as

$$
V(\mathcal{T})=\sum_{\lambda \in \mathbb{Z}} \sum_{\mathcal{N} \in L_{\lambda}} V(\mathcal{N})
$$

Finally, we look for abrupt variance changes that relate to dark areas (i.e. water pixels) or min-tree leaves. We set a threshold value according to this variance change. To illustrate, we plot in Fig. 3 the variance analysis for $\mathcal{T}\left(I_{2}, I_{3}\right)$ for each level $\lambda$, i.e. the inner sum of 3 . We can see there is a turning point close to lowest intensity. We extract all nodes with a level lower than this threshold. 


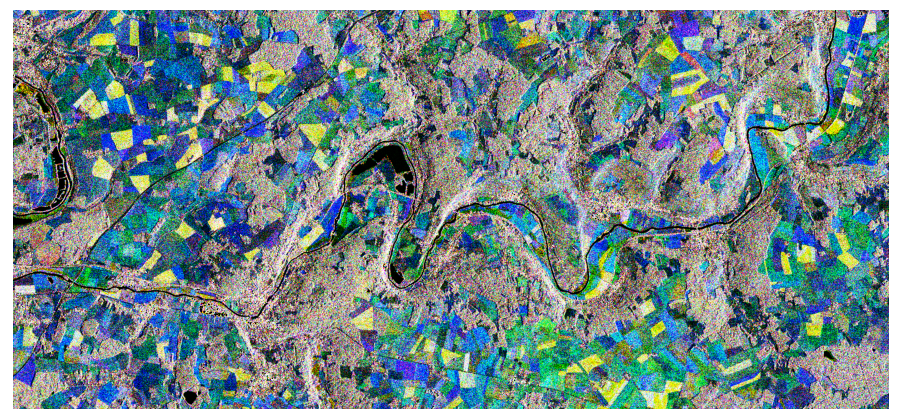

(a) $I$

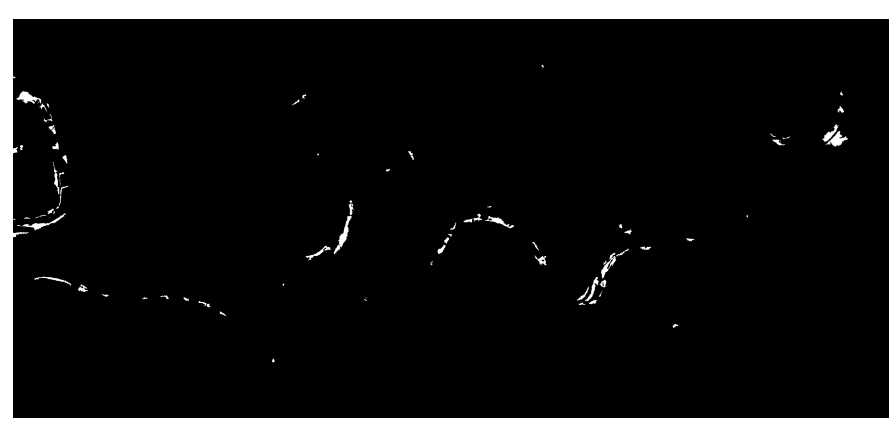

(c) $G T$

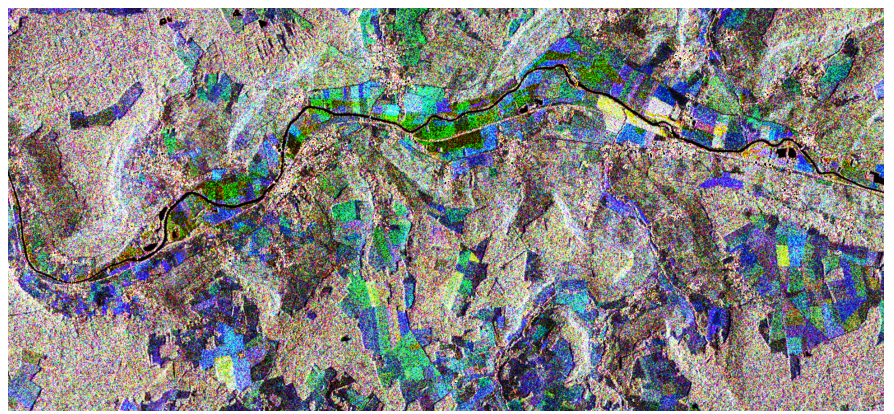

(b) $I^{\prime}$

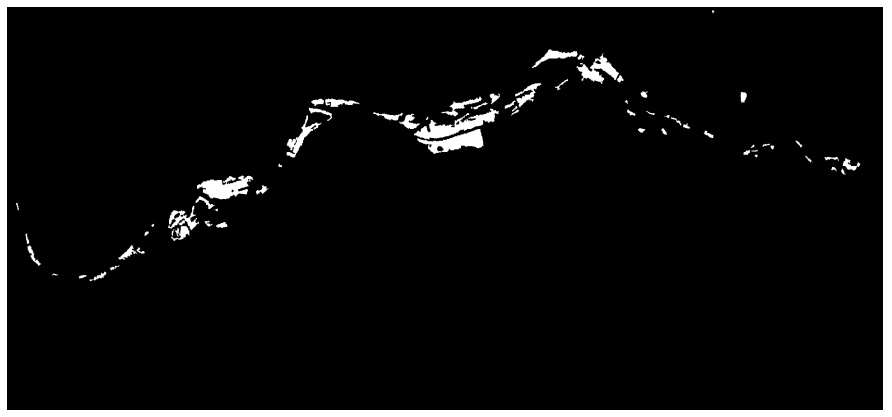

(d) $G T^{\prime}$

Fig. 1: Colorized SITS of two study areas $I$ and $I^{\prime}$, with corresponding reference flood maps.

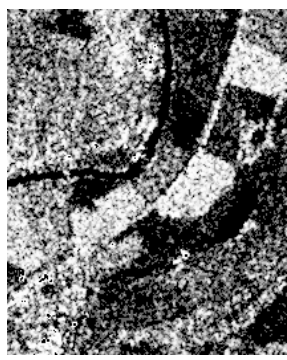

(a) $I_{1}$

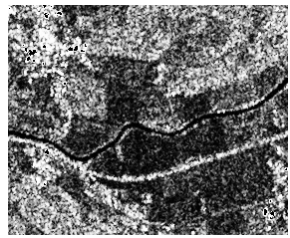

(d) $I_{1}^{\prime}$

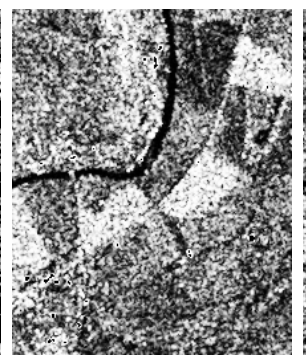

(b) $I_{2}$

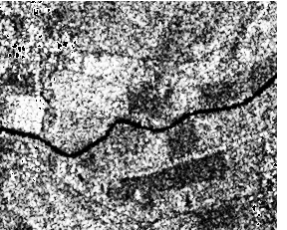

(e) $I_{2}^{\prime}$

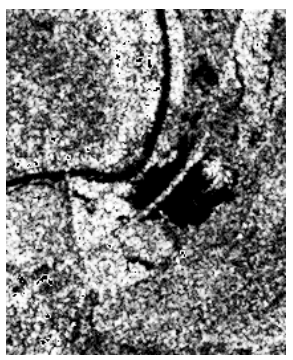

(c) $I_{3}$

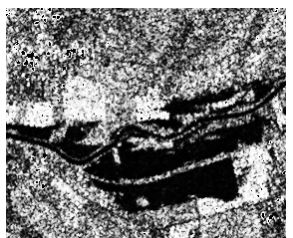

(f) $I_{3}^{\prime}$

Fig. 2: Close-up of the two study areas $I$ and $I^{\prime}$ from Sentinel1 imagery (subscript denotes the temporal index in the SITS).

To further improve robustness to isolated pixels that are most likely to correspond to noise, we also rely on an additional criterion related to the area of the node (i.e. the amount of pixels it contains). We choose here the area threshold $\lambda_{a}$ according to the spatial resolution relationship given in [13], with $\lambda_{a}=\frac{1000}{v}$ where $v$ represents the spatial resolution (in meters) of the input image. We preserve the nodes with an

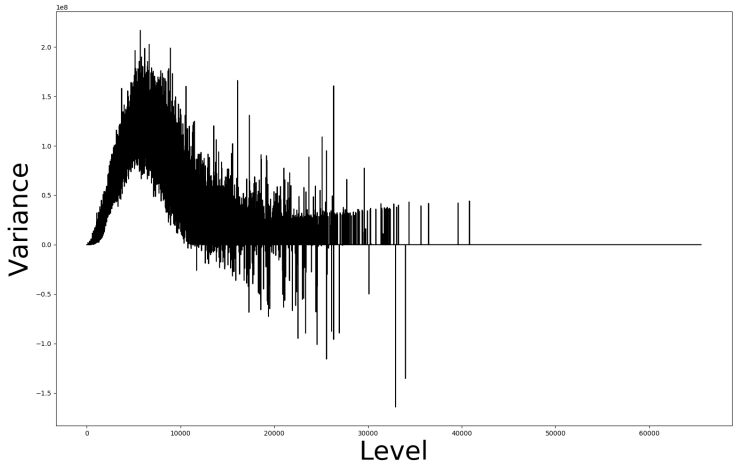

Fig. 3: Total variance difference for each level of the tree.

area larger than this threshold. Since several nested nodes can be kept after this filtering, we remove redundant nodes characterized by a low stability, as given in (1).

At the end of the tree filtering process, the remaining nodes include pixels from the two images used to build the spatiotemporal tree. Since permanent water pixels appear at the same location in the two different images, they belong to the same remaining node in the tree. To distinguish between permanent water areas and floods, we simply reconstruct two binary maps (one per date) from the spatial-temporal tree, before comparing these two maps. Difference between these maps indicate where the floods occur. Nevertheless, we observe that some artifacts [14] can still occur due to double bounce effect, backscatter 


\begin{tabular}{c|c|c|c} 
SITS & Proposed & AP $[8]$ & NDFI [14] \\
\hline$I$ & $\mathbf{0 . 5 5}$ & 0.36 & 0.47 \\
\hline$I^{\prime}$ & $\mathbf{0 . 7 3}$ & 0.51 & 0.60
\end{tabular}

TABLE II: Quantitative evaluation of flood detection for different methods and SITS, using the $F_{1}$ measure.

similarity of dry soil, etc. In order to overcome these errors, we post-process the binary change detection map with small area filtering (e.g. $\lambda_{a}=20$ ).

\section{EXPERIMENTAL RESULTS}

We rely on the open source toolbox iamxt [15] to build the min-tree from the SITS. Figure 4 provides close-ups of colorized SITS (one date per channel) and our results compared with their respective ground truths. In both extracts, our method is able to correctly identify flood areas.

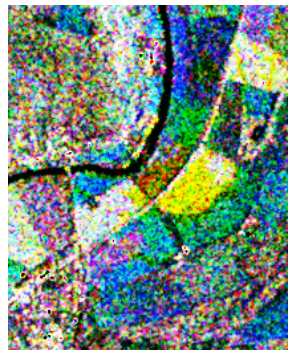

(a) $I$

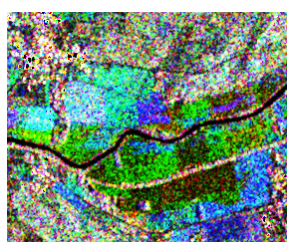

(d) $I^{\prime}$

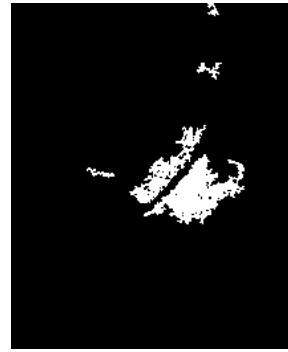

(b) Result

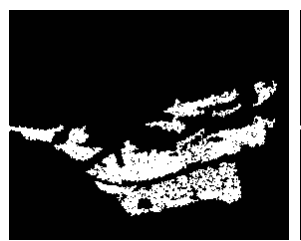

(e) Result

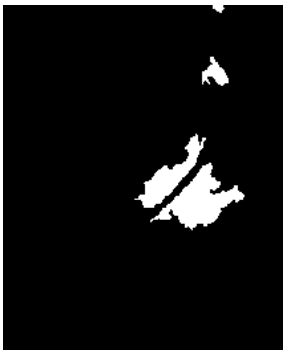

(c) GT

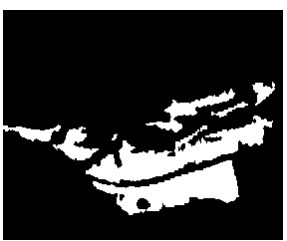

(f) GT
Fig. 4: Close-up of the results (from left to right): color composition of the SITS, comparison between our result and the ground truth (GT).

Furthermore, we report in Table III quantitative results obtained on the whole images from Fig. 1. We consider here the $F_{1}$ score (i.e., the harmonic mean of precision and recall measures). For the sake of comparison, we also report results from two existing methods: AP-based change detection [8] that also relies on spatial attributes extracted from morphological hierarchies, and the Normalized Difference Flood Index (NDFI) [14] thresholding approach. Let us note that the latter is more successful with long time series. We can observe that our method achieves better results and appears as an efficient solution for flood mapping from SAR imagery. Indeed, the whole process is rather efficient, with only 200s per image with an Apple MBP 2.6GHz Intel Core i5 CPU and a Python implementation.

\section{CONCLUSION}

In this paper, we have introduced a novel method for flood detection from SAR imagery. We compute tree-based representations of the SITS to first identify images where floods are most likely to occur, before precisely locating the floods in the scene. Promising quantitative results call for further robustness analysis of the required parameters.

\section{ACKNOWLEDGMENTS}

This work was supported by Centre National d'Études Spatiales (CNES) and Collecte Localisation Satellites (CLS). The authors thank CLS for providing Sentinel-1 images and support in the pre-processing step.

\section{REFERENCES}

[1] A.A. Nielsen, K. Conradsen, H. Skriver, and M.J. Canty, "Change detection in a series of Sentinel-1 SAR data," in MultiTemp, 2017.

[2] S. Martinis, B. Fissmer, and C. Rieke, "Time series analysis of multifrequency SAR backscatter and bistatic coherence in the context of flood mapping," in MultiTemp, 2015.

[3] D. Tang, F. Wang, Y. Xiang, H. You, and W. Kang, "Automatic water detection method in flooding area for GF-3 single-polarization data," in IGARSS, 2018.

[4] P. Bosilj, E. Kijak, and S. Lefèvre, "Partition and inclusion hierarchies of images: A comprehensive survey," Journal of Imaging, vol. 4, no. 2, pp. 33, 2018.

[5] M.T. Pham, S. Lefèvre, E. Aptoula, and L. Bruzzone, "Recent developments from attribute profiles for remote sensing image classification," in ICPRAI, 2018.

[6] A. Alonso-González, C. López-Martínez, and P. Salembier, "Filtering and segmentation of polarimetric sar data based on binary partition trees," IEEE TGRS, vol. 50, no. 2, pp. 593-605, 2012.

[7] A. Alonso-González, C. López-Martínez, and P. Salembier, "Polsar time series processing with binary partition trees," IEEE TGRS, vol. 52, no. 6, pp. 3553-3567, 2014.

[8] N. Falco, M. Dalla Mura, F. Bovolo, J. A. Benediktsson, and L. Bruzzone, "Change detection in vhr images based on morphological attribute profiles," IEEE GRSL, vol. 10, no. 3, pp. 636-640, 2013.

[9] C. Tuna, F. Merciol, and S. Lefèvre, "Monitoring urban growth with spatial filtering of satellite image time series," in JURSE, 2019.

[10] L. Giustarini, R. Hostache, P. Matgen, G.J.P. Schumann, P.D. Bates, and D.C. Mason, "A change detection approach to flood mapping in urban areas using TerraSAR-X," IEEE TGRS, vol. 51, no. 4, pp. 2417-2430, 2013.

[11] P. Salembier, S. Liesegang, and C. López-Martínez, "Ship detection in sar images based on maxtree representation and graph signal processing," IEEE TGRS, 2018.

[12] J. Matas, O. Chum, M. Urban, and T. Pajdla, "Robust wide-baseline stereo from maximally stable extremal regions," Image and Vision Computing, vol. 22, no. 10, pp. 761-767, 2004.

[13] P. Ghamisi, J.A. Benediktsson, G. Cavallaro, and A. Plaza, "Automatic framework for spectral-spatial classification based on supervised feature extraction and morphological attribute profiles," IEEE JSTARS, vol. 7, no. 6 , pp. 2147-2160, 2014.

[14] F. Cian, M. Marconcini, and P. Ceccato, "Normalized difference flood index for rapid flood mapping: Taking advantage of eo big data," Remote Sensing of Environment, vol. 209, pp. 712-730, 2018.

[15] R. Souza, L. Rittner, R. Machado, and R. Lotufo, "iamxt: Max-tree toolbox for image processing and analysis," SoftwareX, vol. 6, pp. 8184, 2017. 